\title{
Bayesian Estimation of Parameters of Weibull Distribution Using Linex Error Loss Function
}

\author{
Josphat. K. Kinyanjui ${ }^{1} \&$ Betty. C. Korir ${ }^{2}$ \\ ${ }^{1}$ Department of Mathematics, Statistics and Actuarial Science, Karatina University, P O Box 1957-10101, Karatina, \\ Kenya \\ ${ }^{2}$ Department of Mathematics and Computer Science, University of Eldoret, P O Box 1125-30100, Eldoret, Kenya \\ Correspondence: Josphat. K. Kinyanjui, Department of Mathematics, Statistics and Actuarial Science, Karatina \\ University, P O Box 1957-10101, Karatina, Kenya. E-mail: jkinyanjui@karu.ac.ke
}

Received: April 18, 2019 Accepted: May 10, 2019 Online Published: February 29, 2020

doi:10.5539/ijsp.v9n2p38 URL: https://doi.org/10.5539/ijsp.v9n2p38

\begin{abstract}
This paper develops a Bayesian analysis of the scale parameter in the Weibull distribution with a scale parameter $\theta$ and shape parameter $\beta$ (known). For the prior distribution of the parameter involved, inverted Gamma distribution has been examined. Bayes estimates of the scale parameter, $\theta$, relative to LINEX loss function are obtained. Comparisons in terms of risk functions of those under LINEX loss and squared error loss functions with their respective alternate estimators, viz: Uniformly Minimum Variance Unbiased Estimator (U.M.V.U.E) and Bayes estimators relative to squared error loss function are made. It is found that Bayes estimators relative to squared error loss function dominate the alternative estimators in terms of risk function.
\end{abstract}

Keywords: Bayes estimates, squared error loss function, LINEX loss function, U.M.V.U.E.

\section{Introduction}

Sometimes, in practical situations either from past experience or from some reliable sources, one may have a guessed estimate of the parameter which can be treated as a prior information. Thompson (1968a, b) introduced the idea of shrinking usual estimators towards point as well as interval guess value to get the improved estimators. In some situations, in place of point estimation or interval guess value, the prior information may be available in the form of prior distribution. Applying Bayesian approach, prior information available in form of prior distribution may be utilized in the estimation of parameters. In Bayesian estimation, the loss function and prior distribution play important role.

The symmetric loss function viz squared error loss function has been widely used by several authors including Berger (1980), Box and Tiao (1973), Martz and Waller (1982), Sinha and Kale (1980). The researchers such as Aitchson and Dunsmore (1975), Berger (1980), Fergusson (1967), Varian (1975) and Zellner and Gielsel (1968), have pointed out that in some situations use of symmetric loss functions may be inappropriate. Actually, we may come across the situations where a given negative error may be more serious than a given positive error or vice-versa, e.g. in dam construction, overestimation of peak of water level is more serious than underestimation. In the same way, in estimation of reliability function, use of symmetric loss function may be inappropriate as recognized by Canfield (1970). Here, overestimation of reliability function or average failure time is more serious than underestimation. [Feymann (1987)]. Varian (1975) proposed a very useful assymetric loss function known as LINEX loss function which rises exponentially on one side of zero and almost linearly on the other side of zero.

Weibull distribution play an important role in many fields of application. It has two parameters $\alpha$ and $\beta$ where ' $\alpha$ ' is referred to as shape parameter and ' $\beta$ ' as scale parameter. This distribution was used by a Swedish scientist Weibull in (1951) to describe experimentally observed variation in fatigue resistance of steel, its elastic limits, e.t.c. It has also been used to study the variation of the length of service of radio service equipment. Finally, it has also been successfully used in reliability theory.

Theoretically, it arises as the limiting distribution as $n \rightarrow \infty$, of the smallest of $\mathrm{n}$ independent random variables with the same distribution. The use of the Weibull distribution as a model analyzing lifetime data, quality and reliability analyses dates back the late nineteenth century. Berger and Sun (1993) gives extensive survey of its uses in the context of lifetime data. Menderhall and Hader (1958) and Cox (1959) are among the first authors who addressed competing risks in survival analysis. Cox (1959) gave other examples where this type of distribution arises. For other references 
concerning competing risks, see David and Moeschberger (1978), Basu and Klein (1982).

Bhattacharya (1967) considered the estimation of both the shape and scale parameters using an inverted Gamma prior probability density function. Canavos and Tsokos (1970) developed a fully Bayesian analysis of both the scale and shape parameters assuming independent prior distributions.

\subsection{LINEX Loss Function}

In some estimation and prediction problems, use of symmetric loss function may be inappropriate, as has been recognized in the literature - see, for example, Ferguson (1967), Zellner and Geisel (1968), Aitchson and Dunsmore (1975), Varian (1975) and Berger (1980).

The authors mentioned above, except for Varian, have considered symmetric linear loss functions. Varian (1975) proposed a very useful assymetric loss function known as LINEX loss function which rises approximately exponentially on one side of zero and almost linearly on the other side of zero in his applied study of real estate assessment. Underassessment results in an approximately linear loss of revenue whereas overassessment often results in appeals with attendant, substantial litigation and other costs.

Attention is directed herein at establishing properties of estimation and prediction procedures based on LINEX loss functions.

Let $\Delta=\hat{\theta}-\theta$ denote the scalar estimation error in using $\hat{\theta}$ to estimate $\theta$. Varian (1975) introduced the following convex loss function:

$$
L(\Delta)=b e^{a \Delta}-c \Delta-b, \quad a, c \neq 0, b>0
$$

It is seen that $L(0)=0$. Also, for a minimum to exist at $\Delta=0$, we must have $a b=c$, and thus equation (2.1) can be reexpressed as

$$
\left.L(\Delta)=b \mid e^{a \Delta}-a \Delta-1\right], \quad a \neq 0, b>0
$$

There are two parameters, $a$ and $b$, involved in equation (2.2) with ' $b$ ' serving to scale the loss function and ' $a$ ' serving to determine its shape.

\subsubsection{Obtaining Bayes Estimators Using Linex Loss Function}

Let $h(\theta / D)$ denote the posterior density function of $\theta$, where $\mathrm{D}$ denotes the sample and prior information, $\left(x_{1}, x_{2}, \cdots, x_{n}, \theta\right)$. Let $\underset{\theta}{E}$ denote the posterior expectation with respect to $h(\theta / D)$. The posterior risk is defined as the $\underset{\theta}{E}[L(\Delta)]$ and is given by

$$
\underset{\theta}{E}[L(\Delta)]=b\left[e^{a \hat{\theta}} \underset{\theta}{E}\left(e^{-a \theta}\right)-a(\hat{\theta}-\underset{\theta}{E}(\theta))-1\right] \ldots
$$

\section{Theorem 2.1}

The value of $\hat{\theta}$ that minimizes $(2.7)$ is

$$
\hat{\theta}_{B}=\frac{-1}{a} \log \left[E\left(e^{-a \theta}\right)\right]
$$

provided, of course, $\underset{\theta}{E}\left(e^{-a \theta}\right)$ exists and is finite. This involves evaluation of moment generating function for posterior density.

\section{Proof}

The conditions for relative minimum are

(i) $\frac{\partial}{\partial \hat{\theta}} \underset{\theta}{E}[L(\Delta)]=0$ and (ii) $\frac{\partial^{2}}{\partial \hat{\theta}^{2}} \underset{\theta}{E}[L(\Delta)]>0$ at the minimum value.

(i) implies that

$$
b\left\lfloor a e^{a \hat{\theta}} E\left(e^{-a \theta}\right)-a\right\rfloor=0 \text { that is } a b\left\lfloor e^{a \hat{\theta}} \underset{\theta}{E}\left(e^{-a \theta}\right)-1\right\rfloor=0
$$

Since $a b \neq 0$, it implies that

$$
e^{a \hat{\theta}} E\left(e^{-a \theta}\right)-1=0
$$


Taking logs and simplifying, we get

$$
\hat{\theta}_{B}=\frac{-1}{a} \log \left[E\left(e^{-a \theta}\right)\right]
$$

(ii) implies that

$$
\begin{aligned}
\frac{\partial^{2}}{\partial \hat{\theta}^{2}} \underset{\theta}{E}[L(\Delta] & =\frac{\partial}{\partial \hat{\theta}}\left[\frac{\partial}{\partial \hat{\theta}} \underset{\theta}{E}[L(\Delta)]\right] \\
& =a^{2} b e^{a \hat{\theta}} \underset{\theta}{E}\left[e^{-a \hat{\theta}}\right]>0
\end{aligned}
$$

Since $\hat{\theta}_{B}$ satisfies conditions (i) and (ii), it follows that $\hat{\theta}_{B}$ is the minimum value.

\section{Bayesian Estimation of Parameters in Case of Weibull Distribution}

\subsection{Introduction}

In this section, we develop a Bayesian analysis for the Weibull distribution with respect to the usual life-testing procedures. It is divided into two parts: in the first, we consider the Bayes estimates of the parameters of Weibull distribution using squared error loss function while in the second part we consider the Bayes estimates of the parameters of Weibull distribution using LINEX error loss function. These estimates obtained based on the two loss functions are later compared in order to show the corresponding efficiencies.

\subsection{Weibull Bayesian Distribution}

The Weibull distribution is given by the probability density function

$$
f(x)=\left\{\begin{array}{l}
\frac{\beta}{\theta} x^{\beta-1} e^{\frac{-x^{\beta}}{\theta}}, 0<x<\infty, \theta, \beta>0 \\
0, \text { Otherwise }
\end{array}\right.
$$

The parameters $\theta$ and $\beta$ are called the scale and shape parameters respectively. Because the shape parameter is known in some cases, we treat the scale parameter $\theta$ as the random variable. We derive a fully Bayesian solution by assuming independent prior distribution of $\theta$. Specifically, we consider an inverted gamma,

$$
\lambda(\theta)=\left\{\begin{array}{l}
\frac{(\mu / \theta)^{v+1} e^{\frac{-\mu}{\theta}}}{\mu \Gamma(v)}, 0<\theta<\infty, \quad \mu, v>0 \\
0, \text { Otherwise }
\end{array}\right.
$$

The reasons for considering the inverted gamma prior density (3.2) are that it is flexible enough to capture almost any kind of prior experience, and it also possesses the attractive property that the posterior distribution of the parameter after the sample has been observed is also of the inverted gamma type. A family of prior densities which gives rise to posteriors belonging to the same family is very useful inasmuch as the mathematical tractability is maintained, and this 'nice' property has been termed 'closure under sampling' by Wetherill (1961). For densities which admit sufficient statistics of fixed dimensionality, Raiffa and Schlaifer have considered a method of generating prior densities on the parameter space that possess this desirable property. A family of such densities has been called by them a 'natural conjugate family', and for Weibull density (3.1), the inverted gamma prior forms such a family.

For our distribution given in (3.1), we have

$$
f(x / \theta, \beta)=L(x, \theta, \beta)=\prod_{i=1}^{n} f\left(x_{i}, \theta, \beta\right)=\left(\frac{\beta}{\theta}\right)^{n} \prod_{i=1}^{n} x_{i}^{\beta-1} e^{\frac{-1}{\theta} \sum_{i=}^{n} x_{i}^{\beta}}
$$

Now substituting the assumed value of $\lambda(\theta)$ given in (3.2) and the value of $f(x / \theta, \beta)$ obtained in (3.3) to (3.1), we obtain 


$$
f(\underline{x}, \theta, \beta)=\frac{\beta^{n} \mu^{v} \prod_{i=1}^{n} x_{i}^{\beta-1} e^{\frac{-1}{\theta} \sum_{i=}^{n}\left(x_{i}^{\beta}+\mu\right)}}{\theta^{n+v+1} \Gamma(v)}
$$

From (3.4), the marginal probability density function of $\mathrm{X}$ is

$$
\begin{aligned}
f(\underline{x}) & =\int_{\theta} f(\underline{x}, \theta, \beta) d \theta=\int_{\theta=0}^{\infty} \frac{\beta^{n} \mu^{v} \prod_{i=1}^{n} x_{i}^{\beta-1} e^{\frac{-1}{\theta} \sum_{i=}^{n}\left(x_{i}^{\beta}+\mu\right)}}{\theta^{n+v+1} \Gamma(v)} d \theta \\
& =\frac{\beta^{n} \mu^{v} \prod_{i=1}^{n} x_{i}^{\beta-1} \Gamma(n+v)}{\left(\mu^{*}\right)^{n+v} \Gamma(v)}
\end{aligned}
$$

where

$$
\mu^{*}=\sum_{i=1}^{n} x_{i}^{\beta}+\mu
$$

The posterior density function of $\theta$ given $X=\underline{x}$ is given by

$$
h(\theta / \underline{x})=\frac{f(\underline{x} / \theta) \lambda(\theta)}{\int_{\theta} f(\underline{x} / \theta) \lambda(\theta) d \theta}
$$

The values obtained in (3.4) and (3.5) are substituted in (3.7) to obtain;

$$
h(\theta / \underline{x})=\frac{\left(\mu^{*}\right)^{n+v} e^{\left(\frac{-1}{\theta}\right) \mu^{*}}}{\theta^{n+v+1} \Gamma(n+v)}
$$

which is the required posterior density function and $\mu^{*}$ is as given in (3.6).

\subsection{Bayes Estimator and Bayes Risk Using Squared Error Loss Function}

\section{Theorem 3.1}

If the loss function is the squared error, $L\left(\theta, \hat{\theta}_{M}\right)=\left(\theta-\hat{\theta}_{M}\right)^{2}$, then the Bayes estimator with respect to the prior distribution $\lambda(\theta)$ of $\theta$ is given by:

$$
\hat{\theta}_{M}=E(\theta / \underline{x})=\frac{\mu^{*}}{n+v-1}
$$

\section{Proof}

From (3.9);

$$
\hat{\theta}_{M}=E(\theta / \underline{x})=\int_{\theta=0}^{\infty} \theta h(\theta / \underline{x}) d \theta
$$

Now substituting the value obtained in (3.8) to (3.10), we get;

$$
\hat{\theta}_{M}=\frac{\left(\mu^{*}\right)^{n+v}}{\Gamma(n+v)} \int_{\theta=0}^{\infty} \frac{e^{\left(\frac{-1}{\theta}\right) \mu^{*}}}{\theta^{n+v+1}} d \theta
$$

which upon simplification gives; 
$\hat{\theta}_{M}=\frac{\mu^{*}}{n+v-1}$ which is the required Bayes estimator of $\theta$.

\section{Theorem 3.2}

If the loss function is the squared error, $L\left(\theta, \hat{\theta}_{M}\right)=\left(\theta-\hat{\theta}_{M}\right)^{2}$, then the corresponding Bayes risk is given by:

$$
{ }_{B} R_{S}\left(\hat{\theta}_{M}\right)=E[\operatorname{Var}(\theta / \underline{x})]=\frac{\left(\mu^{*}\right)^{2}}{(n+v-1)(v-1)(v-2)}
$$

Proof

$$
\operatorname{Var}(\theta / \underline{x})=\int_{\theta}[\theta-E(\theta / \underline{x}, \beta)]^{2} h(\theta / \underline{x}) d \theta=E\left(\theta^{2} / \underline{x}, \beta\right)-[E(\theta / \underline{x}, \beta)]^{2}
$$

Now using (3.8), we get;

$$
E\left(\theta^{2} / \underline{x, \beta}\right)=\int_{\theta=0}^{\infty} \theta^{2} h(\theta / \underline{x}) d \theta=\frac{\left(\mu^{*}\right)^{2}}{(n+v-1)(n+v-2)}
$$

Substituting the values obtained in (3.8) and (3.13) into (3.12), we get;

$$
\operatorname{Var}(\theta / \underline{x})=\frac{\left(\mu^{*}\right)^{2}}{(n+v-1)^{2}(n+v-2)} ; n+v>1
$$

Hence using (3.14) in (3.11), we get;

$$
\begin{aligned}
{ }_{B} R_{S}\left(\hat{\theta}_{M}\right) & =\iint \cdots \int \frac{\beta^{n} \mu^{v} \prod_{i=1}^{n} x_{i}^{\beta-1} \Gamma(n+v)}{(n+v-1)^{2}(n+v-2)\left(\mu^{*}\right)^{n+v-2} \Gamma(v)} d \underline{x} \\
& =\frac{\beta^{n} \mu^{v} \Gamma(n+v)}{(n+v-1)^{2}(n+v-2) \Gamma(v)} \iiint_{x_{1}} \cdots \int \frac{\prod_{i=1}^{n} x_{i}^{\beta-1}}{\left(\mu^{*}\right)^{n+v-2}} d \underline{x}
\end{aligned}
$$

This is simplified to give

$$
{ }_{B} R_{S}\left(\hat{\theta}_{M}\right)=\frac{\beta^{n} \mu^{v} \Gamma(n+v-2)}{(n+v-1) \Gamma(v)} I_{n}
$$

where

$$
I_{n}=\iint_{x_{1} x_{2}} \cdots \int_{x_{n}} \frac{\prod_{i=1}^{n} x_{i}^{\beta-1}}{\left(\sum_{i=1}^{n} x_{i}^{\beta}+\mu\right)^{n+v-2}} d \underline{x}=\frac{\Gamma(v-2)}{\beta^{n} \mu^{v-2} \Gamma(n+v-2)}
$$

Hence from (3.15) and (3.16), we obtain

$$
{ }_{B} R_{S}\left(\hat{\theta}_{M}\right)=\frac{(v-3) ! \mu^{2}}{(n+v-1) \Gamma(v)}=\frac{\mu^{2}}{(v-1)(v-2)(n+v-1)}
$$

which is the required Bayes risk of $\hat{\theta}_{M}$ relative to squared error loss function.

\subsection{Risk Function of $\hat{\theta}_{M}$ Using Linex and Squared Error Loss Function}

In this section, we shall obtain the risk function of the Bayes estimator $\hat{\theta}_{M}$, using both squared and Linex error loss function.

\section{Theorem 3.3}

The risk function of $\hat{\theta}_{M}$ obtained using squared error loss function, $L\left(\theta, \hat{\theta}_{M}\right)=\left(\theta-\hat{\theta}_{M}\right)^{2}$, is given by: 


$$
R_{S}\left(\hat{\theta}_{M}\right)=\left[\frac{\mu-\theta(v-1)}{(n+v-1)}\right]^{2}+\frac{n \theta^{2}}{(n+v-1)^{2}}
$$

\section{Proof}

$$
R_{S}\left(\hat{\theta}_{M}\right)=\iint_{x_{1} x_{2}} \cdots \int_{x_{n}}\left(\hat{\theta}_{M}-\theta\right)^{2} f(\underline{x} / \theta, \beta) d \underline{x}
$$

where $f(\underline{x} / \theta, \beta)$ is the joint distribution.

From (3.3)

$$
\begin{aligned}
& R_{S}\left(\hat{\theta}_{M}\right)=\iint_{x_{1} x_{2}} \cdots \int_{x_{n}}\left(\hat{\theta}_{M}^{2}-2 \theta \hat{\theta}_{M}+\theta^{2}\right) f(\underline{x} / \theta, \beta) d \underline{x} \\
& =\iint_{x_{1}} \cdots \int_{x_{2}} \hat{\theta}_{M}^{2} f(\underline{x} / \theta, \beta) d \underline{x}-2 \theta \iint_{x_{1}} \int_{x_{2}} \cdots \int_{x_{n}} \hat{\theta}_{M} f(\underline{x} / \theta, \beta) d \underline{x}+\theta^{2} \iint_{x_{1}} \int_{x_{2}} \cdots \int_{x_{n}} f(\underline{x} / \theta, \beta) d \underline{x}
\end{aligned}
$$

Substituting the value of $\hat{\theta}_{M}$ obtained in (3.10) to (3.18) we get

$$
\begin{aligned}
& R_{S}\left(\hat{\theta}_{M}\right)=\int_{x_{1}} \cdots \int_{x_{n}}\left(\frac{\mu^{*}}{n+v-1}\right)^{2}\left(\frac{\beta}{\theta}\right)^{n} \prod_{i=1}^{n} x_{i}^{\beta-1} e^{\left(\frac{-1}{\theta}\right) \sum_{i=1}^{n} x_{i}^{\beta}} d \underline{x} \\
& -2 \theta \int_{x_{1}} \cdots \int_{x_{n}}\left(\frac{\mu^{*}}{n+v-1}\right)\left(\frac{\beta}{\theta}\right)^{n} \prod_{i=1}^{n} x_{i}^{\beta-1} e^{\left(\frac{-1}{\theta}\right) \sum_{i=1}^{n} x_{i}^{\beta}} d \underline{x}+\theta^{2}
\end{aligned}
$$

Using $\mu^{*}$ as given in (3.6) in (3.19) gives

$$
\begin{aligned}
& R_{S}\left(\hat{\theta}_{M}\right)=\frac{\beta^{n}}{\theta^{n}(n+v-1)^{2}} \int_{x_{1}} \cdots \iint_{x_{n}}\left(\sum_{i=1}^{n} x_{i}^{\beta}+\mu\right)^{2} \prod_{i=1}^{n} x_{i}^{\beta-1} e^{\left(\frac{-1}{\theta}\right) \sum_{i=1}^{n} x_{i}^{\beta}} d \underline{x} \\
& -\frac{2 \beta^{n}}{\theta^{n}(n+v-1)} \int_{x_{1}} \cdots \int_{x_{n}}\left(\sum_{i=1}^{n} x_{i}^{\beta}+\mu\right) \prod_{i=1}^{n} x_{i}^{\beta-1} e^{\left(\frac{-1}{\theta}\right) \sum_{i=1}^{n} x_{i}^{\beta}} d \underline{x}+\theta^{2}
\end{aligned}
$$

which upon simplification of integrals gives

$$
R_{S}\left(\hat{\theta}_{M}\right)=\left[\frac{\mu-\theta(v-1)}{(n+v-1)}\right]^{2}+\frac{n \theta^{2}}{(n+v-1)^{2}}
$$

\section{Theorem 3.4}

The risk function of $\hat{\theta}_{M}$ using Linex error loss function is given by:

$$
R_{L}\left(\hat{\theta}_{M}\right)=b\left[\frac{e^{-a(\theta-\mu /(n+v-1) /(n+v-1)}}{(1-a \theta /(n+v-1))^{n}}+\frac{a \theta(v-1)-a \mu}{(n+v-1)}-1\right]
$$

\section{Proof}

Using Linex error loss function as given in (2.4), we get

$$
E[L(\Delta)]=b e^{-a \theta} \iint_{x_{1} x_{2}} \cdots \int_{x_{n}} e^{a \hat{\theta}_{M}} f(\underline{x} / \theta) d \underline{x}-a b \iint_{x_{1} x_{2}} \cdots \int_{x_{n}} \hat{\theta}_{M} e^{a \hat{\theta}_{M}} f(\underline{x} / \theta) d \underline{x}+b(a \theta-1)
$$

Substituting the value of $\hat{\theta}_{M}$ obtained in (3.10) to (3.18) we get 


$$
\begin{aligned}
& R_{L}\left(\hat{\theta}_{M}\right)=b e^{-a \theta} \int_{x_{1}} \cdots \int_{x_{n}} e^{a \mu^{*} /(n+v-1)}\left(\frac{\beta}{\theta}\right)^{n} \prod_{i=1}^{n} x_{i}^{\beta-1} e^{\left(\frac{-1}{\theta}\right) \sum_{i=1}^{n} x_{i}^{\beta}} d \underline{x} \\
& -a b \int_{x_{1}} \cdots \int_{x_{n}}\left(\frac{\mu^{*}}{n+v-1}\right)\left(\frac{\beta}{\theta}\right)^{n} \prod_{i=1}^{n} x_{i}^{\beta-1} e^{\left(\frac{-1}{\theta}\right) \sum_{i=1}^{n} x_{i}^{\beta}} d \underline{x}+b(a \theta-1)
\end{aligned}
$$

and using $\mu^{*}$ as given in (3.6), we get

$$
\begin{aligned}
& R_{L}\left(\hat{\theta}_{M}\right)=\frac{b e^{-a \theta} \beta^{n}}{\theta^{n}} \int_{x_{1}} \cdots \int_{x_{n}} e^{a\left(\sum_{i=1}^{n} x_{i}^{\beta}+\mu\right) /(n+v-1)} \prod_{i=1}^{n} x_{i}^{\beta-1} e^{\left(\frac{-1}{\theta}\right) \sum_{i=1}^{n} x_{i}^{\beta}} d \underline{x} \\
& -\frac{a b \beta^{n}}{\theta^{n}(n+v-1)} \int_{x_{1}} \cdots \int_{x_{n}}\left(\sum_{i=1}^{n} x_{i}^{\beta}+\mu\right) \prod_{i=1}^{n} x_{i}^{\beta-1} e^{\left(\frac{-1}{\theta}\right) \sum_{i=1}^{n} x_{i}^{\beta}} d \underline{x}+b(a \theta-1)
\end{aligned}
$$

which when the integrals are solved, the final value is obtained as

$$
R_{L}\left(\hat{\theta}_{M}\right)=b\left[\frac{e^{-a(\theta-\mu /(n+v-1) /(n+v-1)}}{(1-a \theta /(n+v-1))^{n}}+\frac{a \theta(v-1)-a \mu}{(n+v-1)}-1\right]
$$

\section{Theorem 3.5}

The Bayes risk of $\hat{\theta}_{M}$ using Linex error loss function is given by:

$$
\begin{aligned}
& { }_{B} R_{L}\left(\hat{\theta}_{M}\right)= \\
& \frac{b e^{a \mu(n+v-1)} \mu^{(v+2) / 2} a^{v / 2}}{\Gamma(v)}\left[\frac{2}{\mu} K_{v}(2 \sqrt{a \mu}) \frac{2 n}{n+v-1} \sqrt{a / \mu} K_{v-1}(2 \sqrt{a \mu})+\frac{n(n+1) a}{(n+v-1)^{2}} K_{v-2}(2 \sqrt{a \mu})\right]-b
\end{aligned}
$$

where $K_{v}(z)$ is an integral representation of the modified Bessel function of the third kind of order $\mathrm{v}$.

\section{Proof}

The prior risk function of $\hat{\theta}_{M}$ (denoted by $R\left(\lambda, \hat{\theta}_{M}\right)$ ) with respect to the prior distribution $\lambda(\theta)$ of $\theta$ is defined as the prior expectation of the risk function. That is

$$
R\left(\lambda, \hat{\theta}_{M}\right)=E\left[R_{L}\left(\hat{\theta}_{M}\right)\right]=\int_{\theta} R_{L}\left(\hat{\theta}_{M}\right) \lambda(\theta) d \theta
$$

where

$$
R_{L}\left(\hat{\theta}_{M}\right)=E\left[L\left(\hat{\theta}_{M}, \theta\right)\right]
$$

The prior risk function is also called the Bayesian risk or simply the Bayes risk. Thus

$$
{ }_{B} R_{L}\left(\hat{\theta}_{M}\right)=E\left[R_{L}\left(\hat{\theta}_{M}\right)\right]=\int_{\theta=0}^{\infty} R_{L}\left(\hat{\theta}_{M}\right) \lambda(\theta) d \theta
$$

Substituting the values of $\lambda(\theta)$ and $R_{L}\left(\hat{\theta}_{M}\right)$ given in (3.2) and (3.21) respectively to (3.25) we get

$$
\begin{aligned}
& { }_{B} R_{L}\left(\hat{\theta}_{M}\right)=b \int_{\theta=0}^{\infty} \frac{e^{a \mu^{*} /(n+v-1)} e^{-a \theta} \mu^{v} e^{\frac{-\mu}{\theta}}}{[1-a \theta /(n+v-1)]^{n} \theta^{v+1} \Gamma(v)} d \theta-b \int_{\theta=0}^{\infty} \frac{a \mu \mu^{v} e^{\frac{-\mu}{\theta}}}{(n+v-1) \theta^{v+1} \Gamma(v)} d \theta \\
& +\frac{a b(v-1)}{(n+v-1)} \int_{\theta=0}^{\infty} \frac{\theta \mu^{v} e^{\frac{-\mu}{\theta}}}{\theta^{v+1} \Gamma(v)} d \theta-b
\end{aligned}
$$


which further gives;

$$
{ }_{B} R_{L}\left(\hat{\theta}_{M}\right)=\frac{b e^{a \mu^{*} /(n+v-1)} \mu^{v}}{\Gamma(v)} \int_{\theta=0}^{\infty} \frac{e^{-\left(a \theta+\frac{\mu}{\theta}\right)}}{[1-a \theta /(n+v-1)]^{n} \theta^{v+1}} d \theta-b
$$

The integral value is solved as follows:

$$
\int_{\theta=0}^{\infty} \frac{e^{-\left(a \theta+\frac{\mu}{\theta}\right)}}{[1-a \theta /(n+v-1)]^{n} \theta^{v+1}} d \theta=\int_{\theta=0}^{\infty} \frac{e^{-\left(a \theta+\frac{\mu}{\theta}\right)}}{\theta^{v+1}}\left[1-\frac{a \theta}{n+v-1}\right]^{-n} d \theta
$$

Using the binomial expansion to expand $\left[1-\frac{a \theta}{n+v-1}\right]^{-n}$, we have;

$$
\left[1-\frac{a \theta}{n+v-1}\right]^{-n}=1+\frac{n a \theta}{n+v-1}+\frac{n(n+1) a^{2} \theta^{2}}{2(n+v-1)^{2}}+\cdots
$$

To simplify our integration, we can consider the first three terms and assume that the rest are negligible. Since $\frac{a \theta}{n+v-1}<0$

$$
\begin{aligned}
& \int_{\theta=0}^{\infty} \frac{e^{-\left(a \theta+\frac{\mu}{\theta}\right)}}{\theta^{v+1}}\left[1-\frac{a \theta}{n+v-1}\right]^{-n} d \theta=\int_{\theta=0}^{\infty} \frac{e^{-\left(a \theta+\frac{\mu}{\theta}\right)}}{\theta^{v+1}}\left[1+\frac{n a \theta}{(n+v-1)}+\frac{n(n+1) a^{2} \theta^{2}}{2(n+v-1)^{2}}\right] d \theta \\
& =\int_{\theta=0}^{\infty} \frac{e^{-\left(a \theta+\frac{\mu}{\theta}\right)}}{\theta^{v+1}} d \theta+\frac{n a}{(n+v-1)} \int_{\theta=0}^{\infty} \frac{e^{-\left(a \theta+\frac{\mu}{\theta}\right)}}{\theta^{v}} d \theta+\frac{n(n+1) a^{2}}{2(n+v-1)^{2}} \int_{\theta=0}^{\infty} \frac{e^{-\left(a \theta+\frac{\mu}{\theta}\right)}}{\theta^{v-1}} d \theta
\end{aligned}
$$

The above integrals are evaluated by using an integral representation of $K_{v}(z)$, the modified Bessel function of the third kind of order v. (Erd'ely, et. al, formula $23 \mathrm{p} 82$ ), and subsequent use of the same formula in conjunction with the fact that $K_{-v}(z)=K_{v}(z)$. Accordingly,

$$
K_{v}(a z)=\frac{1}{2 a^{v}} \int_{t=0}^{\infty} e^{-\frac{1}{2} z\left(t+\frac{a^{2}}{t}\right)} t^{-v-1} d t
$$

where $\operatorname{Re}(z)>0, \operatorname{Re}\left(a^{2} z\right)>0, \mathrm{z}$ is the variable and $\mathrm{v}$ is the order of the Bessel's function.

The function

$$
K_{v}(z)=\frac{\pi}{2 \sin (v \pi)}\left[\mathrm{I}_{-v}(z)-\mathrm{I}_{v}(z)\right]
$$

is a solution of Bessel differential equation

$$
\begin{gathered}
z^{2} d^{2} w / d z^{2}+z d w / d z-\left(z^{2}+v^{2}\right) w=0 \\
\mathrm{I}_{v}(z)=\sum_{m=0}^{\infty} \frac{x^{2 m+v}}{2^{2 m+v} m ! \Gamma(m+v-1)}
\end{gathered}
$$

Now, in our case;

and by comparing with the relation

$$
\int_{\theta=0}^{\infty} \frac{e^{-\left(a \theta+\frac{\mu}{\theta}\right)}}{\theta^{v+1}} d \theta=\int_{\theta=0}^{\infty} \frac{e^{-a\left(\theta+\frac{\mu}{a \theta}\right)}}{\theta^{v+1}} d \theta
$$




$$
K_{v}\left(a^{*} z\right)=\frac{1}{2 a^{v}} \int_{t=0}^{\infty} \frac{e^{-\frac{1}{2} z\left(t+\frac{a^{2}}{t}\right)} t^{-v-1}}{t^{v+1}} d t
$$

we see that;

$t=\theta, \frac{1}{2} z=a$ which implies that $z=2 a$.

$\left(a^{*}\right)^{2}=\frac{\mu}{a}$ which implies that $a^{*}=\sqrt{\frac{\mu}{a}}$. Therefore;

$$
\int_{\theta=0}^{\infty} \frac{e^{-\left(a \theta+\frac{\mu}{\theta}\right)}}{\theta^{v+1}} d \theta=\left(\frac{2}{\left(a^{*}\right)^{v}}\right) K_{v}\left(a^{*} z\right)=\frac{2}{(\mu / a)^{v / 2}} K_{v}(2 \sqrt{a \mu})
$$

Similarly,

$$
\int_{\theta=0}^{\infty} \frac{e^{-\left(a \theta+\frac{\mu}{\theta}\right)}}{\theta^{v}} d \theta=\frac{2}{(\mu / a)^{(v-1) / 2}} K_{v-1}(2 \sqrt{a \mu})
$$

and

$$
\int_{\theta=0}^{\infty} \frac{e^{-\left(a \theta+\frac{\mu}{\theta}\right)}}{\theta^{v-1}} d \theta=\frac{2}{(\mu / a)^{(v-2) / 2}} K_{v-2}(2 \sqrt{a \mu})
$$

Thus substituting (3.31), (3.32) and (3.33) in (3.30), we obtain

$$
\begin{aligned}
& \int_{\theta=0}^{\infty} \frac{e^{-\left(a \theta+\frac{\mu}{\theta}\right)}}{\theta^{v+1}}\left[1-\frac{a \theta}{n+v-1}\right]^{-n} d \theta=\frac{2}{(\mu / a)^{v / 2}} K_{v}(2 \sqrt{a \mu})+\frac{2 n a}{(n+v-1)(\mu / a)^{(v-1) / 2}} K_{v-1}(2 \sqrt{a \mu}) \\
& +\frac{2 n(n+1) a^{2}}{2(n+v-1)^{2}(\mu / a)^{(v-2) / 2}} K_{v-2}(2 \sqrt{a \mu})
\end{aligned}
$$

Substituting (3.34) in (3.27), we get

$$
\begin{aligned}
& { }_{B} R_{L}\left(\hat{\theta}_{M}\right)= \\
& \frac{b e^{a \mu /(n+v-1)} \mu^{(v+2) / 2} a^{v / 2}}{\Gamma(v)}\left[\frac{2}{\mu} K_{v}(2 \sqrt{a \mu})+\frac{2 n}{n+v-1} \sqrt{a / \mu} K_{v-1}(2 \sqrt{a \mu})+\frac{n(n+1) a}{(n+v-1)^{2}} K_{v-2}(2 \sqrt{a \mu})\right]-b
\end{aligned}
$$

which is the required Bayes risk of $\hat{\theta}_{M}$ using Linex error loss function.

\subsection{Risk Function and Bayes Risk of Uniformly Minimum Variance Unbiased Estimator of $\theta$}

In this section, we shall obtain the Uniformly Minimum Variance Unbiased Estimator of $\theta$. This estimator will be used to obtain the risk function and Bayes risk of the estimator using both squared and Linex error loss function.

\section{Theorem 3.6}

Let $X_{1}, X_{2}, \cdots, X_{n}$ be a random sample of size $\mathrm{n}$ from the Weibull distribution. Let $\hat{\theta}=t\left(x_{1}, x_{2}, \cdots, x_{n}\right)$ be an estimator of $\theta$. Then $\hat{\theta}=\frac{\sum_{i=1}^{n} x_{i}^{\beta}}{n}$ is a unique U.M.V. Unbiased Estimator of $\theta$.

\section{Proof}

The likelihood function of $X_{1}, X_{2}, \cdots, X_{n}$ is given by: 


$$
L(\underline{x}, \theta, \beta)=\prod_{i=1}^{n} f\left(x_{i}, \theta, \beta\right)=\left(\frac{\beta}{\theta}\right)^{n} \prod_{i=1}^{n} x_{i}^{\beta-1} e^{\frac{-1}{\theta} \sum_{i=1}^{n} x_{i}^{\beta}}
$$

Let $t\left(x_{1}, x_{2}, \cdots, x_{n}\right)=\sum_{i=1}^{n} x_{i}^{\beta}$. Hence (3.35) becomes

$L(\underline{x}, \theta, \beta)=g(t, \theta) h\left(x_{1}, x_{2}, \cdots, x_{n}\right)$ where

$g(t, \theta)=\left(\frac{\beta}{\theta}\right)^{n} e^{\frac{-t}{\theta}}$ and $h\left(x_{1}, x_{2}, \cdots, x_{n}\right)=\prod_{i=1}^{n} x_{i}^{\beta-1}$

By factorization theorem, $t\left(x_{1}, x_{2}, \cdots, x_{n}\right)=\sum_{i=1}^{n} x_{i}^{\beta}$ is a sufficient statistics for $\theta$.

To show that $t\left(x_{1}, x_{2}, \cdots, x_{n}\right)=\sum_{i=1}^{n} x_{i}^{\beta}$ is a complete statistics, we need to also show that

$E[h(T)]=0$ implies that $h(T)=0$, for all values of T, where $h(T)$ is a function of T.

$E[h(T)]=\int_{t=0}^{\infty} h(t) \operatorname{Pr}(T=t) d t$ where $T=\sum_{i=1}^{n} x_{i}^{\beta}$

\section{To obtain the p.d.f. of $T$}

$T=\sum_{i=1}^{n} x_{i}^{\beta}$. Let $y=x^{\beta}$ it implies that $x=y^{\frac{1}{\beta}}$ and $\frac{d x}{d y}=\frac{1}{\beta} y^{\frac{1}{\beta}-1}$

Therefore;

$$
g(y)=f(x) \frac{d x}{d y} \text { which reduces to } g(y)=\frac{1}{\theta} e^{\frac{-y}{\theta}}
$$

Since $y_{1}, y_{2}, \cdots, y_{n}$ are independent and identically distributed as $\mathrm{Y}$, then the distribution of $\mathrm{T}$ is;

$$
h(T)=\frac{1}{\theta^{n}} e^{\frac{-t}{\theta}}, \quad 0<t<\infty
$$

$E[h(T)]=0$, then $\sum_{t=0}^{\infty} h(t) \frac{1}{\theta^{n}} e^{\frac{-t}{\theta}}=0$, which on reducing becomes

$$
\left[h(0)+h(1) e^{\frac{-1}{\theta}}+h(2) e^{\frac{-2}{\theta}}+\cdots+h(n) e^{\frac{-n}{\theta}}+\cdots\right]=0
$$

Therefore;

$h(0)=0, h(1)=0, h(2)=0, \cdots, h(n)=0$, that is, $h(t)=0$ for all values of $t=0,1,2, \cdots$

Therefore $T=\sum_{i=1}^{n} x_{i}^{\beta}$ is a complete statistics.

Now;

$$
E(T)=E\left(\sum_{i=1}^{n} x_{i}^{\beta}\right)=\sum_{i=1}^{n} E\left(x_{i}^{\beta}\right)
$$

But; 


$$
E\left(x^{\beta}\right)=\int x^{\beta}\left(\frac{\beta}{\theta}\right)^{n} x^{\beta-1} e^{\frac{-1}{\theta} x^{\beta}} d x=\theta
$$

Thus, (3.36) reduces to

$E(T)=\sum_{i=1}^{n} \theta=n \theta$ and therefore $E(T / n)=\theta$

Thus, $\hat{\theta}=h(T)=\frac{T}{n}=\frac{\sum_{i=1}^{n} x_{i}^{\beta}}{n}$ is the required unique U.M.V. Unbiased Estimator of $\theta$.

\section{Theorem 3.7}

The risk function of $\hat{\theta}$ using squared error loss function is given by $R_{S}(\hat{\theta})=\frac{\theta^{2}}{n}$, where $\hat{\theta}$ is the unique U.M.V. Unbiased Estimator of $\theta$.

\section{Proof}

The risk function of $\hat{\theta}$ is obtained by using the squared error loss function.

$$
R_{S}(\hat{\theta})=E[L(\hat{\theta}, \theta)]=E(\hat{\theta}-\theta)^{2}=\operatorname{Var}(\hat{\theta})
$$

Substituting the value of $\hat{\theta}$ obtained in Theorem 3.6 in (3.37), we get

$$
R_{S}(\hat{\theta})=\operatorname{Var}(\hat{\theta})=\operatorname{Var}\left(\frac{\sum_{i=1}^{n} x_{i}^{\beta}}{n}\right)=\frac{1}{n^{2}} \operatorname{Var}\left(\sum_{i=1}^{n} x_{i}^{\beta}\right)
$$

Since $x_{1}, x_{2}, \cdots, x_{n}$ are independent and identically distributed as $\mathrm{X}$, (3.38) becomes

$$
R_{S}(\hat{\theta})=\frac{n}{n^{2}} \operatorname{Var}\left(x^{\beta}\right)=\frac{1}{n} \operatorname{Var}\left(x^{\beta}\right)
$$

Now we need to obtain $\operatorname{Var}\left(x^{\beta}\right)$.

$$
\text { By definition, } \operatorname{Var}\left(x^{\beta}\right)=E\left(x^{2 \beta}\right)-\left[E\left(x^{\beta}\right)\right]^{2}=E\left(x^{2 \beta}\right)-\theta^{2}
$$

Now;

$$
E\left(x^{2 \beta}\right)=\int x^{2 \beta}\left(\frac{\beta}{\theta}\right)^{n} x^{\beta-1} e^{\frac{-1}{\theta} x^{\beta}} d x=2 \theta^{2} \text { and hence } \operatorname{Var}\left(x^{\beta}\right)=\theta^{2}
$$

The value obtained in (3.41) is substituted in (3.39) to get

$$
R_{S}(\hat{\theta})=\frac{\theta^{2}}{n},
$$

which is the required risk function of the unique U.M.V. Unbiased Estimator of $\theta$.

\section{Theorem 3.8}

The Bayes risk of $\hat{\theta}$ using squared error loss function is given by ${ }_{B} R_{S}(\hat{\theta})=\frac{\mu^{2}}{n(v-1)(v-1)}$, where $\hat{\theta}$ is the unique U.M.V. Unbiased Estimator of $\theta$. 


\section{Proof}

The prior risk function of $\hat{\theta}$ (denoted by $R(\lambda, \hat{\theta})$ ) with respect to the prior distribution $\lambda(\theta)$ of is defined as the prior expectation of the risk function. That is

$$
R(\lambda, \hat{\theta})=E\left[R_{S}(\hat{\theta})\right]=\int R_{S}(\hat{\theta}) \lambda(\theta) d \theta
$$

The prior risk function is also called the Bayesian risk or simply the Bayes risk

Thus;

$$
{ }_{B} R_{S}(\hat{\theta})=\int_{\theta=0}^{\infty} R_{S}(\hat{\theta}) \lambda(\theta) d \theta
$$

The values of $\lambda(\theta)$ and $R_{S}(\hat{\theta})$ given in (3.2) and in Theorem 3.7 respectively are used in (3.43) to obtain

$$
{ }_{B} R_{S}(\hat{\theta})=\int_{\theta=0}^{\infty} R_{S}(\hat{\theta}) \lambda(\theta) d \theta=\int_{\theta=0}^{\infty} \frac{\theta^{2}}{n} \cdot \frac{\left(\frac{\mu}{\theta}\right)^{v+1} e^{\frac{-\mu}{\theta}}}{\mu \Gamma(v)} d \theta=\frac{\mu^{v+1}}{n \mu \Gamma(v)} \int_{\theta=0}^{\infty}\left(\frac{1}{\theta}\right)^{v-1} e^{\frac{-\mu}{\theta}} d \theta
$$

which after simplification becomes

$$
{ }_{B} R_{S}(\hat{\theta})=\frac{\mu^{v+1}}{n \mu \Gamma(v)} \cdot \frac{\Gamma(v-2)}{\mu^{v-2}}=\frac{\mu^{2}}{n(v-1)(v-2)} \text { as required. }
$$

\section{Theorem 3.9}

The risk function of $\hat{\theta}$ using Linex error loss function is given by $R_{L}(\hat{\theta})=b\left[\frac{e^{-a \theta}}{\left(1-\frac{a \theta}{n}\right)^{n}}-1\right]$, where $\hat{\theta}$ is the unique U.M.V. Unbiased Estimator of $\theta$.

\section{Proof}

Using Linex error loss function as given in (2.4), we get

$$
\begin{aligned}
& R_{L}(\hat{\theta})=b e^{-a \theta}\left(\frac{\beta}{\theta}\right)^{n} \int_{x_{1}} \cdots \int_{x_{n}} e^{\frac{a}{n}\left(\sum_{i=1}^{n} x_{i}^{\beta}\right)} \prod_{i=1}^{n} x_{i}^{\beta-1} e^{\left(\frac{-1}{\theta}\right) \sum_{i=1}^{n} x_{i}^{\beta}} d \underline{x} \\
& -\frac{a b \beta^{n}}{n \theta^{n}} \int_{x_{1}} \cdots \int_{x_{n}} \sum_{i=1}^{n} x_{i}^{\beta} \prod_{i=1}^{n} x_{i}^{\beta-1} e^{\left(\frac{-1}{\theta}\right) \sum_{i=1}^{n} x_{i}^{\beta}} d \underline{x}+b(a \theta-1)
\end{aligned}
$$

Using integration by parts to integrate the integral

$$
\int_{x_{1}} \cdots \int_{x_{n}} \prod_{i=1}^{n} x_{i}^{\beta-1} e^{-\sum_{i=1}^{n} x_{i}^{\beta}\left[\frac{1}{\theta}-\frac{a}{n}\right]} d \underline{x}, \text { we obtain } \int_{x_{1}} \cdots \int_{x_{n}}^{n} \prod_{i=1}^{n} x_{i}^{\beta-1} e^{-\sum_{i=1}^{n} x_{i}^{\beta}\left[\frac{1}{\theta}-\frac{a}{n}\right]} d \underline{x}=\left[\frac{n \theta}{\beta(n-a \theta)}\right]^{n}
$$

Similarly, using integration by parts to integrate the integral

$$
\int_{x_{1}} \cdots \int_{x_{n}} \sum_{i=1}^{n} x_{i}^{\beta} \prod_{i=1}^{n} x_{i}^{\beta-1} e^{-\frac{1}{\theta} \sum_{i=1}^{n} x_{i}^{\beta}} d \underline{x}, \text { we get } \int_{x_{1}} \cdots \int_{x_{n}} \sum_{i=1}^{n} x_{i}^{\beta} \prod_{i=1}^{n} x_{i}^{\beta-1} e^{-\frac{1}{\theta} \sum_{i=1}^{n} x_{i}^{\beta}} d \underline{x}=\frac{n \theta^{n+1}}{\beta^{n}}
$$

Hence (3.45) and (3.46) are used in (3.44) to obtain; 


$$
R_{L}(\hat{\theta})=b e^{-a \theta}\left(\frac{\beta}{\theta}\right)^{n}\left[\frac{n \theta}{\beta(n-a \theta)}\right]^{n}-\frac{a b \beta^{n}}{n \theta^{n}}\left[\frac{n \theta^{n+1}}{\beta^{n}}\right]+b(a \theta-1)
$$

which reduces to

$$
R_{L}(\hat{\theta})=b\left[\frac{e^{-a \theta}}{\left(1-\frac{a \theta}{n}\right)^{n}}-1\right]
$$

Theorem 3.10

The Bayes risk of $\hat{\theta}$ using Linex error loss function is given by ${ }_{B} R_{L}(\hat{\theta})=\frac{b \mu^{(v+2) / 2} a^{(v-2) / 2}}{\Gamma(v)}\left[2\left(\frac{a}{\mu}\right) K_{V}(2 \sqrt{a \mu})+2 a \sqrt{\frac{a}{\mu}} K_{V-1}(2 \sqrt{a \mu})+\frac{(n+1) a^{2}}{n} K_{V-2}(2 \sqrt{a \mu})\right]-b$, where $\hat{\theta} \quad$ is the unique

U.M.V. Unbiased Estimator of $\theta$.

\section{Proof}

The prior risk function is also called the Bayesian risk or simply the Bayes risk. Thus

$$
{ }_{B} R_{L}(\hat{\theta})=E\left[R_{L}(\hat{\theta})\right]=\int_{\theta=0}^{\infty} R_{L}(\hat{\theta}) \lambda(\theta) d \theta
$$

Substituting the values of $\lambda(\theta)$ and $R_{L}(\hat{\theta})$ given in (3.2) and Theorem (3.9) respectively to (3.47) we get

$$
{ }_{B} R_{L}(\hat{\theta})=b \int_{\theta=0}^{\infty}\left[\frac{e^{-a \theta}}{[1-a \theta / n)]^{n}}-1\right] \frac{(\mu / \theta)^{v+1} e^{\frac{-\mu}{\theta}}}{\mu \Gamma(v)} d \theta=\frac{b \mu^{v}}{\Gamma(v)} \int_{\theta=0}^{\infty} \frac{e^{-(a \theta+\mu / \theta)}}{\theta^{v+1}}\left[1-\frac{a \theta}{n}\right]^{-n} d \theta-b
$$

The integral is solved as in (3.27) which then gives the final value of the integral as;

$$
{ }_{B} R_{L}(\hat{\theta})=\frac{b \mu^{(\nu+2) / 2} a^{(v-2) / 2}}{\Gamma(v)}\left[2\left(\frac{a}{\mu}\right) K_{V}(2 \sqrt{a \mu})+2 a \sqrt{\frac{a}{\mu}} K_{V-1}(2 \sqrt{a \mu})+\frac{(n+1) a^{2}}{n} K_{V-2}(2 \sqrt{a \mu})\right]-b
$$

\section{Computation of Relative Efficiency and Comparison in Terms of Risk Functions}

\subsection{Introduction}

In this section, we make comparisons of the obtained estimators in terms of risk functions of those under Linex loss and squared error loss function. Once Bayes estimators under Linex loss function and squared error loss function have been obtained, comparisons in terms of their risk functions have been made, their relative efficiencies are computed. Thus some conclusions based on computations and graphs regarding relative efficiencies for some effective intervals will help us to know what estimators performs better than alternative estimators in terms of effective interval relative to Linex loss function than those relative to squared error loss function.

\subsection{Computation of Relative Efficiencies and Comparison in Terms of Risk Function of Weibull Bayesian Distribution}

In this section, we will use some of the results obtained section three. We have obtained that the risk functions, denoted by $R_{S}\left(\hat{\theta}_{M}\right), R_{L}\left(\hat{\theta}_{M}\right), R_{S}(\hat{\theta})$ and $R_{L}(\hat{\theta})$, where the subscript L denotes risk relative to Linex error loss function and $\mathrm{S}$ denotes risk relative to squared error loss function. These risk functions are given in Theorems 3.3, 3.4, 3.7, and 3.9 respectively.

Let us define relative efficiencies of the estimator $\hat{\theta}_{M}$ with respect to $\hat{\theta}$ under the Linex and squared error loss function as follows:

$$
R E_{L}\left(\hat{\theta}_{M}, \hat{\theta}\right)=R_{L}(\hat{\theta}) / R_{L}\left(\hat{\theta}_{M}\right) \text { and } R E_{S}\left(\hat{\theta}_{M}, \hat{\theta}\right)=R_{S}(\hat{\theta}) / R_{S}\left(\hat{\theta}_{M}\right)
$$


These relative efficiencies (RE) are functions of $\mathrm{a}, \mu, \theta$, n, and v. For some sets of values of a, $\mu, \theta, \mathrm{n}$, and v, the graphs of the relative efficiencies, plotted against $\theta$ are shown in Figs. 4.1 and 4.2.

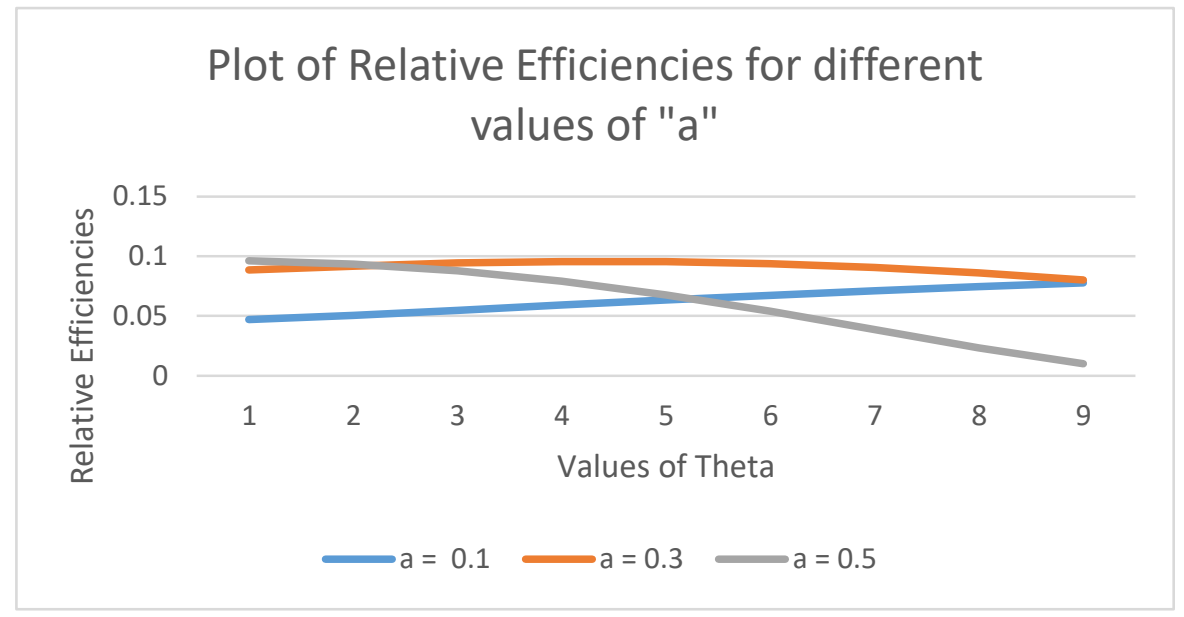

Figure 4.1. Plot of $R E_{L}\left(\hat{\theta}_{M}, \hat{\theta}\right)$ for different values of "a" given $\mathrm{n}=4, \mu=3$ and $\mathrm{v}=0.5$

From the above figure, we observe that for an increase in the magnitude of "a", ttytytyty

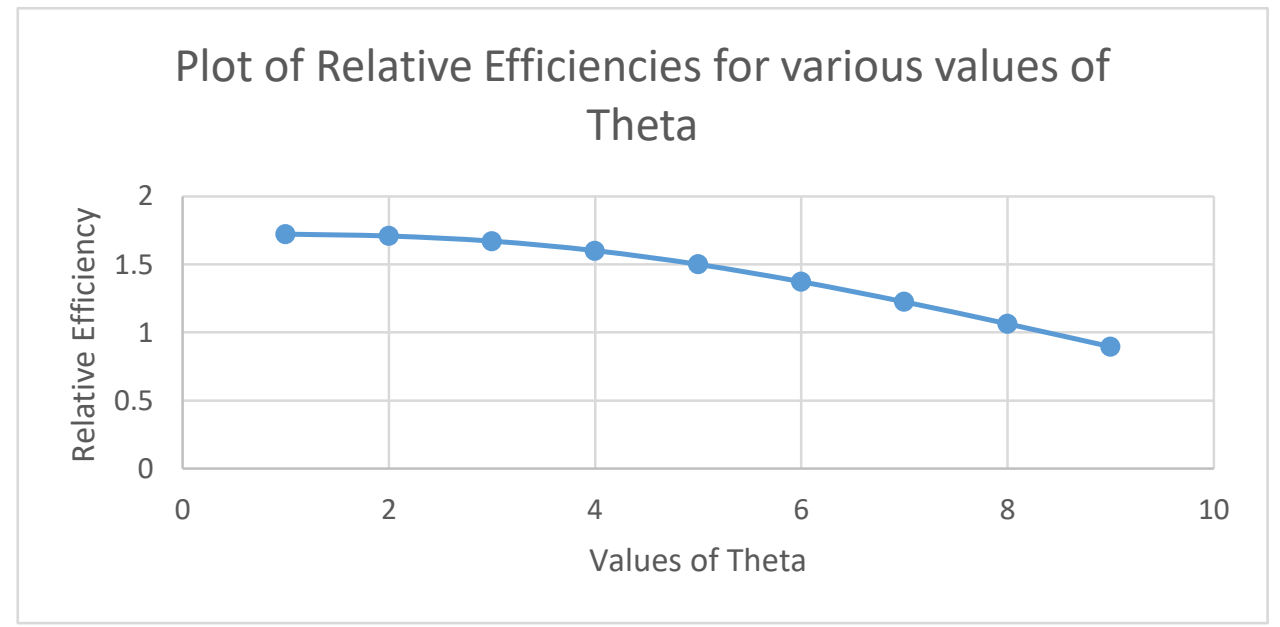

Figure 4.2. Plot of $R E_{S}\left(\hat{\theta}_{M}, \hat{\theta}\right)$ for different values of "Theta" given $\mathrm{n}=4, \mu=3$ and $\mathrm{v}=0.5$

From the above figure, for an increase in the values of $\theta$, there is a decrease in the magnitude of RE.

Thus some conclusions based on graphs regarding effective interval reveals that for the Weibull distribution, $\hat{\theta}_{M}$ performs better than alternative estimators in terms of effective interval relative to squared error loss function than those relative to Linex error loss function.

\section{Summary and Concluding Remarks}

Asymmetric LINEX loss functions have been employed in the analysis of several central statistical estimation and prediction problems. Optimal estimators and predictors relative to LINEX loss and their associated risk functions have been derived. The analytical ease with which results can be obtained using asymmetric LINEX loss functions makes them attractive for use in applied problems and in assessing the effects of departures from assumed symmetric loss functions. For example, Pandey and Rai (1992), in the normal - mean problem, found that Bayes estimators relative to LINEX loss functions dominate the alternative estimators in terms of risk function and Bayes risk. They also found out that if $\sigma^{2}$ is unknown, the Bayes estimators are still preferable over alternative estimators. In the Weibull distribution, it was straight forward to derive an estimator that is optimal relative to LINEX loss function and to obtain its risk function and Bayes risk. Also, certain well known estimators, for example, the U.M.V. Unbiased Estimator of $\theta$, that are admissible relative to squared error loss function were shown to be also admissible relative to LINEX loss function. As a referee Zellner (1973) has stated, “...the point that questions of admissibility may depend quite sensitively on 
features of the loss function, such as symmetry, is not generally appreciated..." and implies that a lot more thought should be given to the choice of a loss function, rather than to blindly trust in squared error loss function", see Zellner (1973) for an analysis of the effects of errors in specifying loss functions on solutions to control problems. While the LINEX class of loss functions is convenient and useful, it is recognized that other asymmetric loss functions, for example, asymmetric linear and quadratic loss functions, are available and may be useful. Further study of the properties of alternative estimators relative to these and other types of asymmetric loss functions would be useful and is left for future research.

\section{References}

Aitchison, J., \& Dunsmore, I. R. (1975). Statistical Prediction Analysis, London: Cambridge University Press. https://doi.org/10.1017/CBO9780511569647

Alexander, M. M. Introduction to the Theory of Mathematical Statistics, Third ed. McGraw - Hill series in Probability and Statistics.

Basu, A. P., \& Ebrahim, N. (1988). Bayesian Approach to Life Testing and Reliability Estimation Using Asymmetric Loss Function. Mathematical Science Technical Report No. 144. AFOSSR, Technical Report No. 88.

Basu, A. P., \& Klein, J. P. (1982). Some recent results in competing risks theory. Lecture Notes-Monograph Series, 2 , 216-229. https://doi.org/10.1214/lnms/1215464851

Berger and Sun (1993). Bayesian Analysis for the Poly - Weibull Distribution. JASA, 88(424). https://doi.org/10.1080/01621459.1993.10476426

Berger, J. O. (1980). Statistical Decision Theory: Foundations, Concepts and Methods, New - York Springer - Verlag. https://doi.org/10.1007/978-1-4757-1727-3_1

Bhattacharya, S. K. (1962). On a point Analogue used in a Life Test based on Weibull Distribution, Australia Journal of Statistics, 4, 101-105. https://doi.org/10.1111/j.1467-842X.1962.tb00327.X

Bhattacharya, S. K. (1966). A Modified Bessel Function Model in Life Testing. Metrika, 11, $133-144$. https://doi.org/10.1007/BF02613584

Bhattacharya, S. K. (1967). Bayesian Approach to Life Testing and Reliability Estimation. JASA, 62(317), 48-62. https://doi.org/10.1080/01621459.1967.10482887

Bhattacharya, S. K., \& Holla, M. S. (1965). On a Discrete Distribution with Special Reference to the Theory of Accident Proneness. JASA, 60, 1060-1066. https://doi.org/10.1080/01621459.1965.10480850

Canavos and Tsokos (1970). Bayesian Estimation of Life Parameters in the Weibull Distribution. Operations Research, 45, 24-31.

Canfield, R. V. (1970). A Bayesian Approach to Reliability Estimation Using a Loss Function, I.E.E.E. Transaction on Reliability, 19, 13-16. https://doi.org/10.1109/TR.1970.5216372

Cohen, A. C. (1965), Maximum Likelihood Estimation in the Weibull Distribution based on complete and Censored Samples, Technometrics, 7, 579-588. https://doi.org/10.1080/00401706.1965.10490300

Cox, D. R. (1959). The Analysis of Exponentially Distributed Lifetimes with two types of Failures. The Journal of Royal Statistical Society, Ser. B., 21, 411-421. https://doi.org/10.1111/j.2517-6161.1959.tb00349.x

David, H. A., \& Moeschberger, M. L. (1978). The Theory of Competing Risks. Griffin's Statistical Monographs and Courses, No. 39, London: Charles. W. Griffin.

Drake, A. W. (1996). Bayesian Statistics for the reliability Engineer, Proc. Annual Symposium on reliability, $315-320$.

Ehrenfeld, S. (1962). Some Experimental Design Problems in Life Testing. JASA, 57, 668-679. https://doi.org/10.1080/01621459.1962.10500555

Epstein, B., \& Sobel, M. (1953). Life Testing. JASA, 48, 486-502. https://doi.org/10.1080/01621459.1953.10483488

Erdelyi, A. et.al. (1953). Higher Transcedental Functions. Vol. II, McGraw Hill Book Company, inc. New - York.

Ferguson, T. S. (1967). Mathematical Statistics: A decision Theoretical Approach, New - York: Academic Press.

Hogg, R. V., \& Craig, A. T. (1956). Introduction to mathematical statistics, Pg.200-227.

Mendehall, W. (1958). A Bibliography on Life Testing and Related Topics. Biometrika, 45, 521-543. https://doi.org/10.1093/biomet/45.3-4.521

Mendenhall, W., \& Hadel, R. J. (1958). Estimation of Parameters of Mixed Exponentially Distributed Failure Time 
Distributions from Censored Life Test Data. Biometrika, 45, 504-520. https://doi.org/10.1093/biomet/45.3-4.504

Pandey, \& Rai. (1992), Bayesian Estimation of Mean and Square of Mean of Normal Distribution using LINEX Loss Function, Communication in Statistics, 21(12), 3369-3391. https://doi.org/10.1080/03610929208830985

Searls, D. T. (1964). The Utilization of a Known Coefficient of Variation in the Estimation Procedure. JASA, 59, 1225-1226. https://doi.org/10.1080/01621459.1964.10480765

Sinha, S. K., \& Kale, B. K. (1980). Life Testing and Reliability Estimation, Wiley Eastern Limited, New Delhi.

Tate, R. F. (1959). Unbiased Estimation: Functions of Location and Scale Parameters. Ann. Math. Stat., 30, $341-366$. https://doi.org/10.1214/aoms/1177706256

Thompson, J. R. (1968). Some Shrinkage Techniques for Estimating the Mean. JASA, 63, 113-123. https://doi.org/10.2307/2283832

Thompson, J. R. (1968b). Accuracy Borrowing in the Estimation of Mean by Shrinkage to an Interval. JASA, 63, 953-963. https://doi.org/10.1080/01621459.1968.11009322

Varian, H. R. (1975). A Bayesian approach to real estate assessment. Studies in Bayesian econometric and statistics in Honor of Leonard J. Savage, 195-208.

Wetherill, G. B. (1961), Bayesian Sequential Analysis, Biometrika, 48, $281-292$. https://doi.org/10.1093/biomet/48.3-4.281

Zellner, A. (1971).An Introduction to Bayesian Inference in Econometrics, New - York, John Willey and sons, inc.

Zellner, A. (1973). The quality of Quantitative Economic Policymaking When Targets and Costs of Change are Misspecified. In Selected Readings in Econometrics and Economic Theory: Essays in Honour of Jan Tinbergen, ed. W. Sellekaerts, London: Macmillan, 147-164. https://doi.org/10.1007/978-1-349-01936-6_7

Zellner, A. (1986), Bayes Estimation and Prediction Using Asymmetric Loss Functions. JASA, 81, 446-451. https://doi.org/10.1080/01621459.1986.10478289

\section{Copyrights}

Copyright for this article is retained by the author(s), with first publication rights granted to the journal.

This is an open-access article distributed under the terms and conditions of the Creative Commons Attribution license (http://creativecommons.org/licenses/by/4.0/). 